OPEN ACCESS

Edited by:

Marc Pallardy,

Université Paris-Sud, France

Reviewed by:

Rami Bechara,

University of Pittsburgh,

United States

Saadia Kerdine-Römer,

Université Paris-Sud, France

*Correspondence:

Samuel Ken-En Gan

Samuel_Gan@eddc.a-star.edu.sg;

samgan@apdskeg.com

${ }^{\dagger}$ These authors have contributed

equally to this work

Specialty section: This article was submitted to B Cell Biology,

a section of the journal

Frontiers in Immunology

Received: 04 March 2021 Accepted: 24 May 2021

Published: 08 July 2021

Citation:

Su CT-T, Lua W-H, Poh J-J, Ling W-L, Yeo JY and Gan SK-E (2021) Molecular Insights of Nickel Binding to Therapeutic Antibodies as a Possible New Antibody Superantigen.

Front. Immunol. 12:676048.

doi: 10.3389/fimmu.2021.676048

\section{Molecular Insights of Nickel Binding to Therapeutic Antibodies as a Possible New Antibody Superantigen}

\author{
Chinh Tran-To Su ${ }^{1+}$, Wai-Heng Lua ${ }^{1 \dagger}$, Jun-Jie Poh ${ }^{1}$, Wei-Li Ling ${ }^{1}$, Joshua Yi Yeo ${ }^{1}$ \\ and Samuel Ken-En Gan ${ }^{1,2,3 *}$ \\ ${ }^{1}$ Antibody \& Product Development Lab, Experimental Drug Development Centre, Bioinformatics Institute, Agency for \\ Science, Technology, and Research (A*STAR), Singapore, Singapore, 2 James Cook University, Singapore, Singapore, \\ ${ }^{3}$ APD SKEG Pte Ltd, Singapore, Singapore
}

The binding of nickel by immune proteins can manifest as Type IV contact dermatitis (Nispecific $T$ cells mediated) and less frequently as Type I hypersensitivity with both mechanisms remaining unknown to date. Since there are reports of patients comanifesting the two hypersensitivities, a common mechanism may underlie both the TCR and IgE nickel binding. Focusing on Trastuzumab and Pertuzumab IgE variants as serendipitous investigation models, we found Ni-NTA interactions independent of Her2 binding to be due to glutamine stretches. These stretches are both Ni-inducible and in fixed pockets at the antibody complementarity-determining regions (CDRs) and framework regions (FWRs) of both the antibody heavy and light chains with influence from the heavy chain constant region. Comparisons with TCRs structures revealed similar interactions, demonstrating the possible underlying mechanism in selecting for Ni-binding IgEs and TCRs respectively. With the elucidation of the interaction, future therapeutic antibodies could also be sagaciously engineered to utilize such nickel binding for biotechnological purposes.

Keywords: allergy, glutamine, type I hypersensitivity, IgG1, IgE, nickel (II), antibody

\section{INTRODUCTION}

Nickel (Ni) is ubiquitous in household products and food (1), and can cause allergic reactions for $\sim 15 \%$ of the population (2) making it one of the most common metal allergies (3). It can manifest as either type IV contact dermatitis mediated by hapten-specific T cells (2) and very occasionally as IgE-mediated type I hypersensitivity (4). Associations between the two allergies are increasingly reported $(5,6)$ where they can occur within the same patient $(4)$. The similarities in the structural topologies between the antibody variable $(\mathrm{V})$ regions (complementarity-determining regions or CDRs and framework regions or FWRs) with those of T cell receptor (TCR) may provide a clue to a possible common pathogenesis (7-9) from the clonal selection and expansion of such Ni reacting Tand B-cells. Known to be shuttled by albumin and other nickel binding proteins (10), metal-protein complexes formed with nickel can activate T-cells and also cross-link nickel-specific IgEs (11). Thus, 
the elucidation of the mechanism in one immune protein (e.g. IgE) may also provide insight into TCR activation which can in itself play the dual role in selectively expanding type IV and type I hypersensitivity effector cells, where activated CD4+ cells can also support the IgE producing B-cells via linked recognition (12).

Histidine binding of $\mathrm{Ni}^{2+}$ ions is long-known and has been exploited in his-tag protein affinity purification (13). This also underlies the interaction of the major histocompatibility complex (MHC) with certain peptides (14, 15), Hpn-like protein (16), in Fc $\gamma$ (17), and in TCRs of type IV contact dermatitis patients (18). In biologics, this mechanism may partly account for the dermatological adverse effects associated with certain targeted therapies (19-21), where apart from directly causing type I and IV responses, the metal binding can also contribute to protein aggregation (22), increasing immunogenicity (23).

Ni IgE-mediated hypersensitivity occurs most commonly as occupational hazards (24), where the prolonged exposures elicited Ni-interacting IgEs (25) through possible "nonantigenic" mechanisms (26) that arise from the expansion of existing IgEs (capable of additionally binding $\mathrm{Ni}$ ) producing $\mathrm{B}$ cells. We hypothesize the Ni-only specific IgEs to be extremely rare given the chemical nature of $\mathrm{Ni}$, but rather that the unintended non-specific binding to $\mathrm{Ni}$ during antibody repertoire generation to other antigens occurred and were gradually selected for and expanded. To investigate this possible mechanism, a holistic investigation (27) of whole IgEs to study the various antibody regions and their possible combination to structurally form histidine-rich Ni-binding patches are required. This likelihood has support from previous findings showing that different antibody regions interact synergistically in antigen binding $(28,29)$ and FcR engagement $(30,31)$.

While superantigens are classically defined to directly bind and activate TCRs (32), their classifications have been expanded to include microbial proteins that bind to immunoglobulins, termed B-cell (33) or Ig/antibody superantigens e.g. Proteins A and L (34). These antibody superantigens can bind specifically to antibody regions without being recognized as conventional antigens and are often used for purification. Despite having Tcell superantigen-like properties, nickel was excluded as a superantigen on the basis of requiring specific interactions as with conventional TCR superantigens (35). Nonetheless, this exclusion may be challenged when applied to antibody superantigens where the known protein L superantigen interaction with antibody FWR1 can be affected by deletions in FWR3 while largely keeping the equivalent VH-VL pairing (36).

Nonetheless, since the binding of both TCR- and antibodies can arise from a common superantigen-like mechanism, an indepth molecular investigation is warranted.

Fortuitously, our recombinant Trastuzumab and Pertuzumab IgE VH-FWR swapped variants (30) were found to bind Ni-NTA without diminishing antigen (Her2) specificity. It is from this panel that we sought to unravel the binding mechanism of $\mathrm{Ni}$ to the IgEs.

\section{MATERIALS AND METHODS}

\section{Cell Culture}

HEK EXPI293F (GIBCO) were grown in high glucose Dulbecco's Modified Eagle Medium (DMEM, GIBCO) with 1X PenicillinStreptomycin (Nacalai Tesque), $2 \mathrm{mM}$ L-Glutamine (Biological Industries), and $10 \%$ heat-inactivated FBS (GIBCO), at $37^{\circ} \mathrm{C}$ and $5 \% \mathrm{CO}_{2}$.

\section{Antibody Production and Purification}

Pertuzumab/Trastuzumab IgE VH family and Pertuzumab/

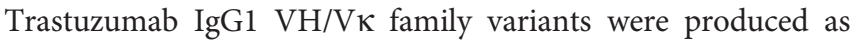
previously described $(30,31)$. Pertuzumab and Trastuzumab

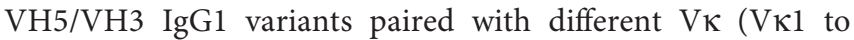
$\mathrm{V \kappa 6}$ ) were produced in the same manner. A protein L column was used for IgE purification, while a protein $\mathrm{G}$ column was used for IgG1.

\section{Bio-Layer Interferometry Studies and Cross Metal Reactivity}

Association, dissociation, and equilibrium dissociation rate constants of the Igs to the Ni-NTA were measured as was performed previously $(30,31)$ through direct binding of the antibodies to NTA biosensors (Fortebio) recharged with $10 \mathrm{mM}$ $\mathrm{NiCl} 2$ for $1 \mathrm{~min}$ on the Octet Red96 system (Fortebio).

For cross metal reactivity measurements, Ni-NTA biosensors were stripped with low pH $1.710 \mathrm{mM}$ glycine and recharged with $10 \mathrm{mM}$ of Cobalt (II) Chloride $\left(\mathrm{CoCl}_{2}\right)$, Nickel (II) Chloride $\left(\mathrm{NiCl}_{2}\right)$, or Copper (II) Chloride $\left(\mathrm{CuCl}_{2}\right)$, before binding with $100 \mathrm{nM}$ of Pertuzumab VH5 IgE variants.

\section{Modeling Trastuzumab and Pertuzumab VH3, VH5, and VH7 IgE and VH5 IgG1}

Models of Pertuzumab and Trastuzumab VH3, VH5, and VH7 IgEs paired with their respective $\mathrm{V} \kappa 1$ were constructed previously (30). The Pertuzumab VH5-Vк1 IgG1 model was built via displacement of the Ce of Pertuzumab VH5-Vк1 IgE structure with $\mathrm{C} \gamma$ (IgG1) followed by minimization and equilibration (1 ns, explicit solvent with sequential constant volume and pressure, NVT and NPT ensembles, respectively)

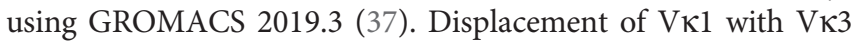
and Vк6 of the Pertuzumab and Trastuzumab VH5 IgG1 gave rise to the other models.

\section{Molecular Dynamics (MD) Simulation of Trastuzumab and Pertuzumab IgE ${ }^{\mathrm{Fab}}$ and $\lg \mathbf{F}^{\mathrm{Fab}}$}

To maintain the neutral charges and avoid interference at the two termini of each single Fab domain, the Fab models of whole Pertuzumab and Trastuzumab IgE and IgG1 variants were extracted and capped with ACE/NME at the N- and Cterminus respectively. The system was then relaxed by short minimization (5000 steps) and heated in gradual thermal baths from $0-100 \mathrm{~K}$ (with constant volume) and then from 100-300 K (with constant pressure). The system was then equilibrated (1 ns) 
and followed by $100 \mathrm{~ns}$ production applying explicit solvent model in triplicates $(3 \times 100 \mathrm{~ns})$. The MD simulations were carried out with random velocities and constrained by the Langevin temperature equilibration scheme to thermalize the systems at $300 \mathrm{~K}$ at time steps of $2 \mathrm{fs}$, using AMBER14 (38).

\section{Blind Docking of Nickel $\left(\mathrm{Ni}^{2+}\right)$-Bound NTA Onto the Pertuzumab and Trastuzumab VH3, VH5, and VH7 IgE ${ }^{\mathrm{Fab}}$}

The Ni-bound NTA (nitrilotriacetic acid) was first constructed using topologies of NTA (from PDB: 1GVC) and of $\mathrm{Ni}^{2+}$ ion (from PDB: 4PPT). AM1-BCC charge method (implemented in antechamber) was applied using Chimera (39) to compute partial charges of the constructed Ni-bound NTA, termed Ni-NTA.

Blind dockings using Autodock Vina (40) with Ni-NTA (as ligand) and the Pertuzumab and Trastuzumab VH3, VH5, or VH7 IgE Fab domains (C€2 onwards were assumed to be insignificant, thus excluded) were performed. Ni-NTA internal bonds were kept rigid to minimize confounding binding to the respective Fabs while also constraining the flexibility of the free $\mathrm{Ni}^{2+}$ from binding to the deeply buried cavities. A $1-\AA$ grid box was placed at the molecule center and extended to cover the whole Fab domain. Pre-defined parameters of $\mathrm{Ni}^{2+}$ ion in the AutodockTools packages were used. The dockings were performed for 10 replicates that represent 10 different $\operatorname{IgE}^{\mathrm{Fab}}$ conformations of each variant, resulting with $10 \times 1000$ conformers for each and with exhaustive searching set at 128. A distance cut-off of $4 \AA$ between the $\mathrm{Ni}^{2+}$ ion of the NiNTA and the interacting residue was used to determine the binding.

\section{Cryptic Pocket Detection}

Using an online server CryptoSite (41), cryptic binding site detection was performed on the Fab domains of Pertuzumab

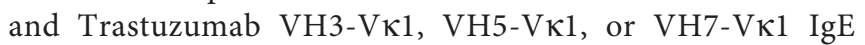
variants. The predicted cryptic residues were then matched into identified pockets resulting from 'mdpocket' (42). Only internal (hidden) pockets or open channels (with isovalue $\geq 5$ ) detected in more than $80 \%$ of the MD trajectories were selected and classified as dense (isovalue $\geq 8$ ) or less dense ( $5 \leq$ isovalue $<$ 8 ) cavities. Pockets were deemed cryptic if they contained at least one cryptic residue predicted by the CryptoSite.

\section{Structural Analysis of Protein-Metal Complexes}

Protein-metal complexes of $\mathrm{Ni}^{2+}, \mathrm{Cu}^{2+}$, and $\mathrm{Co}^{2+}$ from Protein Data Bank (PDB, accessed in June 2020) were enquired using the advance ligand search function with keywords: 'NI', 'CU', or 'CO' in the "Identifier" search. Only protein complexes containing standalone metal ligands were retrieved. The search was further narrowed to immune protein complexes by extending the keywords to "immune", "antibody", "MHC”, and “TCR".

\section{Statistical Analysis}

All KD (M), ka (1/Ms), and kd (1/s) measurements were performed in at least duplicates at concentrations from 200
$\mathrm{nM}$ to $3.125 \mathrm{nM}$ of the antibodies using the Octet RED96 system and analysis software. Measurement responses of less than $0.1 \mathrm{~nm}$ for at least three concentrations were classified as immeasurable or "poor responses".

\section{RESULTS}

\section{The Role of VH on Additional Ni-Binding}

Recombinant Pertuzumab and Trastuzumab IgE and IgG variants (Figure S1) of $\mathrm{VH}$ families: VH1 to $\mathrm{VH} 7$ were paired

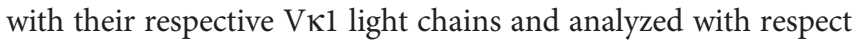
to nickel-attached nitrilotriacetic acid biosensor (Ni-NTA) binding using the Octet RED96 bio-layer interferometry system. Measurements using NTA alone (without $\mathrm{Ni}^{2+}$ ) with Pertuzumab VH5 IgE ruled out NTA as the interacting ligand (Figure S2), providing confidence that experiments measured only Ni interactions.

All Pertuzumab IgE VHx variants (except for VH3 and VH6) interacted with the Ni-NTA sensor (determined by equilibrium dissociation constants $\mathrm{KD}$ ), with the VH5 variant having the lowest KD (best binding, shown in Figure 1A). For Pertuzumab IgG1 variants, all but VH5 had poor responses in the measurements (Figure 1B), indicating that the swapping of heavy chain constant region $\mathrm{Ce}$ (IgE) to $\mathrm{C} \gamma$ (of IgG1) ablated the Ni-NTA interactions. Conversely, the swap of $\mathrm{Ce}$ to $\mathrm{C} \gamma$ improved Ni-NTA interactions for Trastuzumab VH3 and VH5 variants, thereby showing (e.g., in the Pertuzumab data) that the $\mathrm{CH}$ contributed to Ni-binding at the $\mathrm{V}$-regions.

There were varying Ni-NTA KD measurements within the Pertuzumab and Trastuzumab VH variants (Figure 1). Using Pertuzumab CDRs, VH5 IgE and IgG1 had the best binding (KD $\sim 5.30 \mathrm{nM}$ and $\sim 14.73 \mathrm{nM}$, respectively) among all the Pertuzumab variants. When grafted with Trastuzumab CDRs, both the VH3 and VH7 IgE and IgG1 variants as well as VH5 IgG1 yielded lower KD (better binding) while VH5 IgE showed a reverse trend. We could not test the other Trastuzumab $\mathrm{VH}$ variants as they could not be produced as previously mentioned (30).

\section{$\mathrm{Ni}^{2+}$ Binding Glutamines}

While $\mathrm{Ni}^{2+}$ binding to histidine $(\mathrm{H})$ is canon (43), the noncanonical glutamine $(\mathrm{Q})$ could also bind $\mathrm{Ni}^{2+}$ as observed for HLA-DR52C (44) and in a single domain antibody VHH (45). Analysis of $8,054 \mathrm{Ni}^{2+}$ interactions ( $0.4 \%$ of $\mathrm{Q}$ and $59.2 \%$ of $\mathrm{H}$ ) from 1,810 protein-Ni (II) complexes (from PDB in June 2020) revealed that structural stretches of residues accounted for the Ni binding.

The $\mathrm{H}$ and $\mathrm{Q}$ residue counts within both Pertuzumab and Trastuzumab V-regions showed no observable pattern (Table S1). Blind dockings with Ni-NTA of the VH3, VH5, and VH7 Pertuzumab and Trastuzumab IgE Fab regions (containing VHx$\mathrm{V \kappa} 1, \mathrm{C} \kappa$, and $\mathrm{C} \epsilon 1)$ showed interactions at the glutamine (Q) stretches (Figure $\mathbf{2}$ and Figure S3). Specifically, Ni-NTA interacted with the Q-stretch involving residues Q38 (Vк1 FWR2) and Q39 (VH3, VH5, and VH7 FWR2) of the 

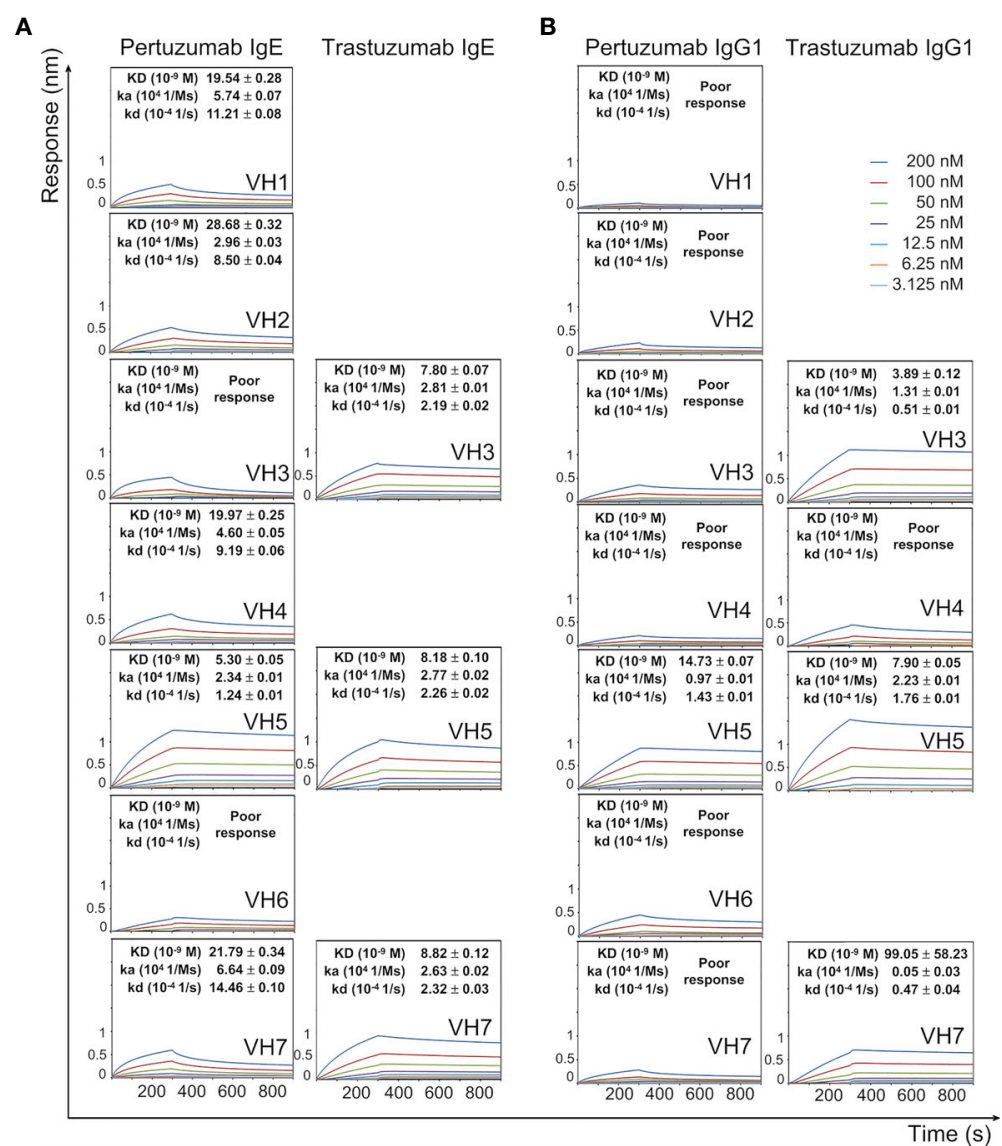

FIGURE 1 | Dissociation equilibrium constants (KD) of Pertuzumab and Trastuzumab IgE (A) and lgG1 (B) of different VH families with the Ni-NTA biosensor. The measurements were performed at concentrations from $200 \mathrm{nM}$ to $3.125 \mathrm{nM}$ of the antibodies. Values of KD (M), ka (1/Ms), and kd (1/s) were determined using the Octet RED96 system. The X-axis depicts the time (in seconds) while the Y-axis depicts the binding responses (nm). All the experiments were conducted in at least duplicates. "Poor response" reflects measurement responses that are less than $0.1 \mathrm{~nm}$ for at least three concentrations. Variants that could not be produced are left empty.

Trastuzumab IgE variants (Figure S3) with Q37 of Vк1 FWR2. This Q-stretch is found in a stable ( $3 \times 100$ ns simulation trajectories) cryptic pocket at the interface of the heavy and light chains (cyan in Figure 2A, see methods and Table S2) in Trastuzumab but not Pertuzumab IgE, Instead, a lower density cavity formed in Pertuzumab VH7 IgE by Q39 (VH7 FWR2), Q37 and Q38 of the $\mathrm{V} \kappa 1$ (yellow in Figure 2A). Agreeing with experimental measurements where Pertuzumab VH7 IgE binds Ni-NTA poorer than Trastuzumab VH7 IgE (Figure 1A), a lower populated NiNTA cluster occurred at Q39 of the VH7 FWR2 as opposed to the corresponding area for Trastuzumab VH7-Vк1 IgE (Figure S3).

The lack of Q-stretch pockets within Pertuzumab VH3-Vк1

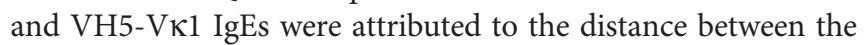
buried VH Q39 residue from Vא1 Q37 and Q38 (Figures 2A, B). Ni-interactions by VК1 Q89, VH5 H67 (in FWR3), and heavy chain CDR2 D50 in both VH3 and VH5 of Pertuzumab IgEs were also found (Figure S3). Interestingly, H67 of VH5-FWR3 is present in both Trastuzumab and Pertuzumab models suggesting its importance in the $\mathrm{Ni}$ engagement.

Site-directed H67Q mutagenesis (in VH5-10 and other VH5 germlines) of both Pertuzumab and Trastuzumab VH5 IgEs disrupted the Ni binding, having pronounced effects in Pertuzumab $\mathrm{VH} 5^{\mathrm{H} 67 \mathrm{Q}} \operatorname{IgE}(\sim 5$-fold $\mathrm{KD}$ increase $)$ and less in Trastuzumab $\mathrm{VH}^{\mathrm{H} 67 \mathrm{Q}} \operatorname{IgE}(\sim 1.5$-fold $\mathrm{KD}$ increase, Figure 3). Although $\mathrm{H} 67$ is present in the VH5 FWR3 of both antibodies, it is only in Pertuzumab VH5 that the residue synergized with Vк1 Q89 for Ni-binding. This was contrary to the Q39-Q37/Q38 stretch in Trastuzumab VH5 that played the key role of $\mathrm{Ni}$ engagement (Figure 3).

The poor responses in Pertuzumab VH3 IgE Ni engagement (Figure 1A) agreed with the blind docking results showing general scattering with denser congregation around D50 of the VH CDR2 (Figure S3). This D50 formed a polar contact network extending to residue 67 of the VH FWR3, i.e., R67 and H67 of Pertuzumab VH3 and VH5 IgE, respectively (Figure 2C). When stable, the D50-R67 interaction decreased Ni-NTA interaction whereas its absence in Pertuzumab VH5 IgE permitted greater Ni-NTA accessibility.

\section{The Role of $\mathbf{V}_{\kappa}$ in Ni-Binding}

To study the role of $\mathrm{V} \kappa$, Pertuzumab and Trastuzumab V $\kappa 1$ to $\mathrm{V} \kappa 6$ light chains were paired with their respective $\mathrm{VH} 3$ and $\mathrm{VH} 5$ (lowest KD in Ni-NTA binding in Figure 1B) IgG1 variants. 

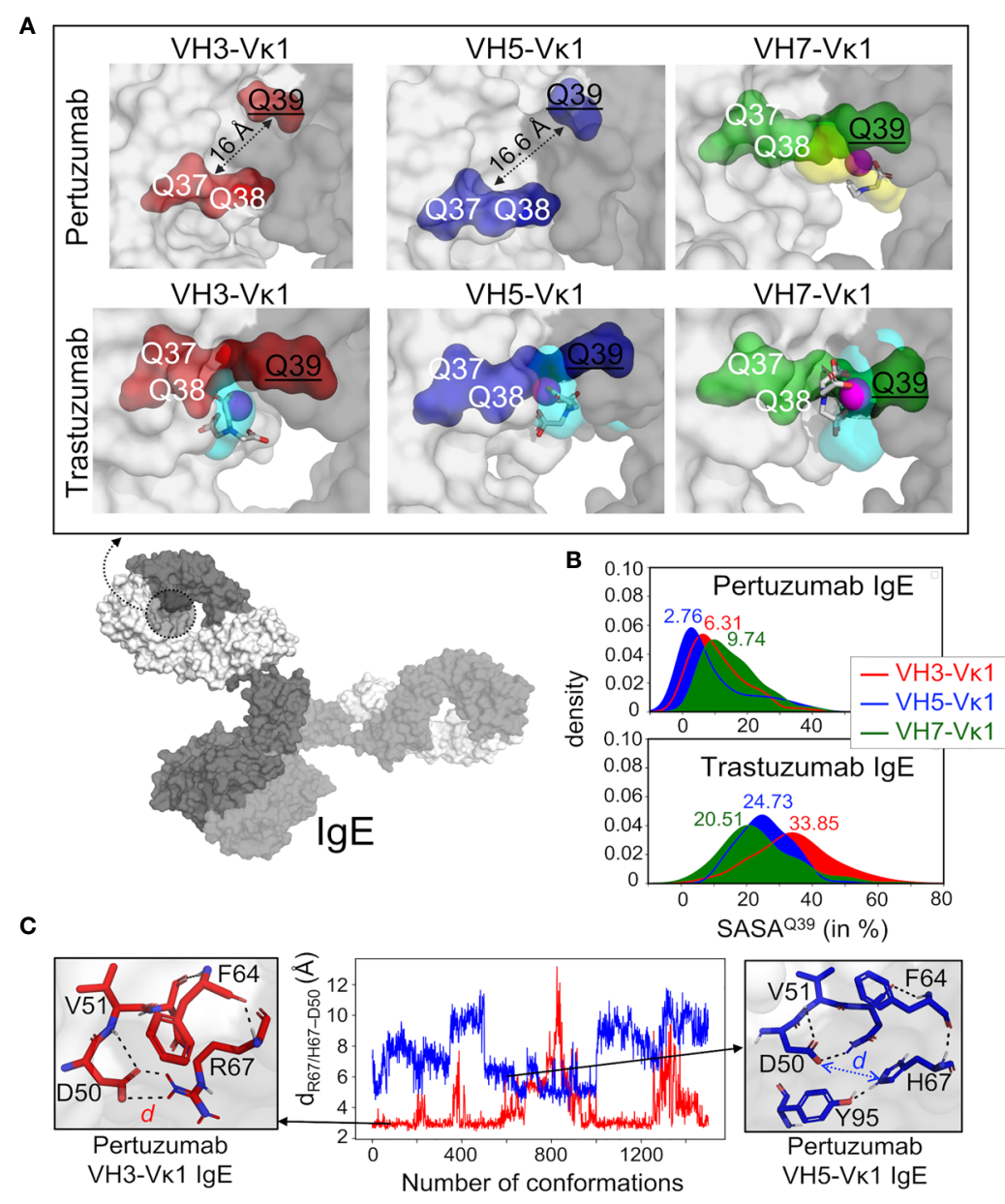

FIGURE 2 | Structural analyses of glutamine from Pertuzumab and Trastuzumab VH3, VH5, and VH7 lgE variants in binding Ni-NTA. (A) Model of whole lgE (L-chain in white surface and $\mathrm{H}$-chains in gray surface) reveals the formed pocket at the VHx-VK1 interface (dashed circle). The Ni-NTA bound region of the variants VH3 (red), VH5 (blue), and VH7 (green) shows the glutamine (Q) stretch constituted by the VH Q39 (underlined) and Vא1 Q37-Q38 to form a stable cryptic pocket (capacity of which is shown in cyan transparent surface) in the Trastuzumab IgE variants or a transient non-cryptic pocket (yellow transparent surface) in the Pertuzumab VH7. The bound Ni-NTA is shown in magenta spheres and interacts at the pocket in both Pertuzumab and Trastuzumab. (B) Relative solvent accessible surface area (SASA) of the Pertuzumab and Trastuzumab VH Q39 residue in all the variants to demonstrate the deeply buried Q39 (SASA<10\%) of the Pertuzumab variants. (C) Distance $d$ between residue 67 (R67 in VH3 or H67 in VH5) and H-chain CDR2 D50 of Pertuzumab VH3-Vk1 lgE (red) and VH5-Vk1 lgE (blue) during the simulation. Structural visualizations were generated using PyMOL 2.3.2 (46). An augmented reality (AR) presentation of the Ni-NTA binding to Trastuzumab VH5 IgE glutamine-forming pocket could be viewed using our "APD AR Holistic Review" app available on both Google and Apple app stores [for more details see ref $(47,48)$ ].

With the exception of VK5 that was not produced, all Pertuzumab VH3 IgG1 variants of the various VKs showed poor Ni-NTA responses (Figure 4A). When Vк1 was replaced

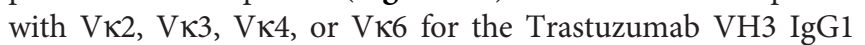
variants, the Ni-NTA bindings could not be measured, although a higher KD was found with VK5 (increase from $3.89 \mathrm{nM}$ to $\sim 12.83 \mathrm{nM}$ ).

The VH5 IgG1 variants of both Pertuzumab and Trastuzumab had stronger Ni engagement than their VH3 counterparts (Figure 4B). This was especially so for

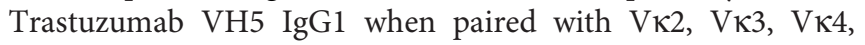
and Vк6 FWRs. Of the VH5 variants (except for the unproduced Vא5), Pertuzumab IgG1s that were paired with VК3 and Vк6 could not be measured (Figure 4B). Since sequence analysis showed only differences in the heavy/light CDRs between Pertuzumab and Trastuzumab VH5 IgG1, stronger Trastuzumab interaction is attributed to the CDRs. As

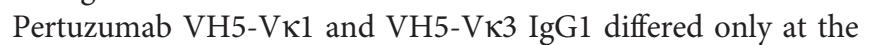
light chain FWRs, the reverse binding trend demonstrated the synergistic contribution of both FWRs and CDRs of both heavy and light chains.

Analysis of the Q and $\mathrm{H}$ residue counts between the Vк family variants (Table S1) showed relative consistency for both Pertuzumab and Trastuzumab VH5 IgG1 variants. Distance-

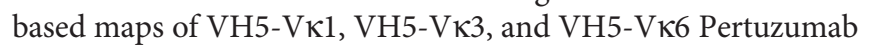
and Trastuzumab $\operatorname{IgG1}^{\mathrm{Fab}}$ variants showed that $\mathrm{V} \kappa$ changes caused conformational changes while retaining $\mathrm{VH} 5, \mathrm{C} \gamma 1$, and

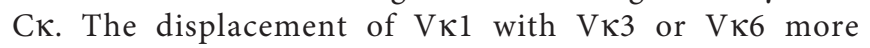


A

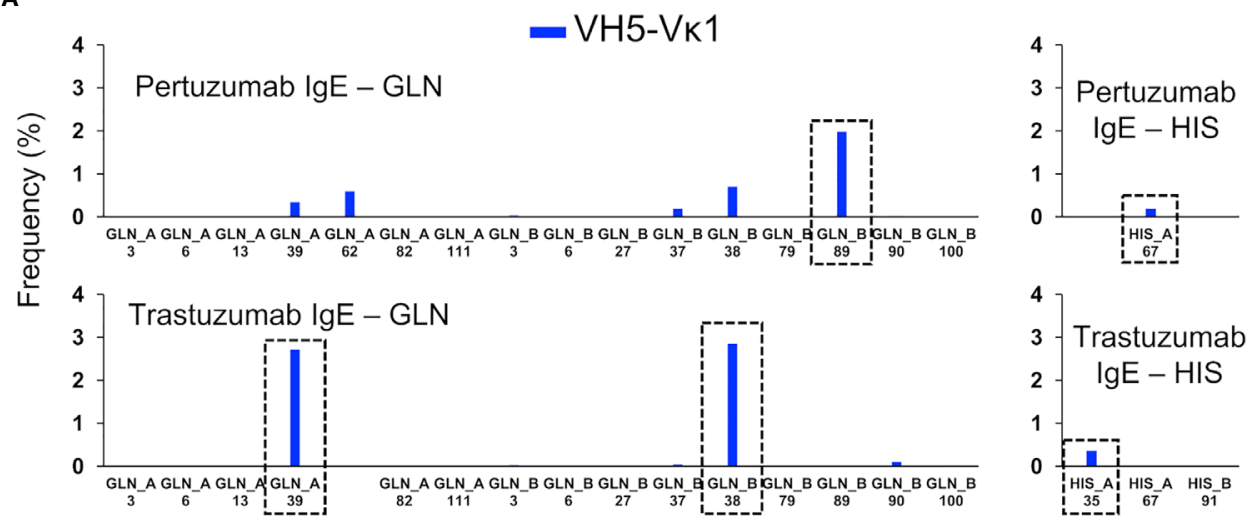

B

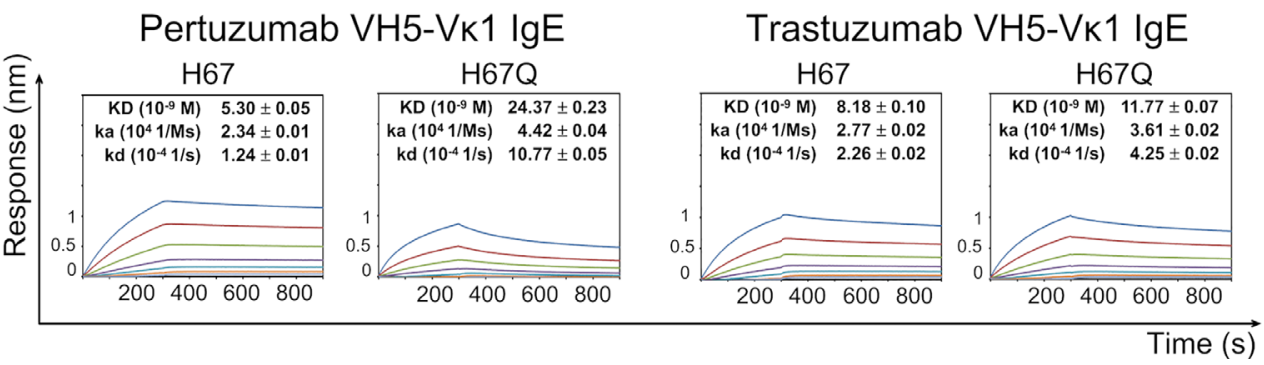

FIGURE 3 | The Ni-NTA binding region of the Pertuzumab VH5-VK1 IgE. The main residue for the interaction is H67 of VH5 FWR3, whereas for Trastuzumab VH5VK1 lgE, it is at the Q-stretch containing VH5 Q39 at FWR2. (A) Distribution of the Ni-NTA docked conformers showing all the glutamine (GLN) and histidine (HIS) present in the $\mathrm{V}$-region of the Pertuzumab and Trastuzumab VH5-Vk1 lgE variants. The predominant binding residue locations are highlighted in dashed boxes. (B) KD of the Pertuzumab and Trastuzumab VH5-VK1 IgE variants including H67Q mutation.

pronouncedly disrupted Pertuzumab VH5 IgG1 variants (red color in Figure 5A) with respect to the $\mathrm{C} \kappa$ accommodation.

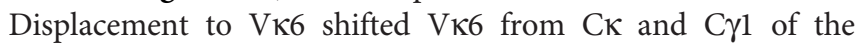
Pertuzumab IgG1 variants. In comparison, smaller effects were

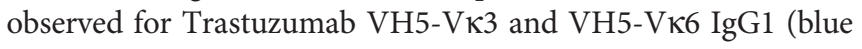
and green in Figure 5A).

Blind dockings of Ni-NTA to the Trastuzumab VH5-Vк1, VH5$\mathrm{V \kappa 3}$, and VH5-Vк6 IgG1 Fab revealed Ni-NTA interactions at the Q-stretch of VH5 Q39 and VК Q37-Q38 of the heavy-light chain interface, as also observed for Trastuzumab VH5-Vк1 IgE (Figures S3 and S4). Given that the heavy and light chain geometry of the Trastuzumab VH5 $\operatorname{IgG} 1^{\mathrm{Fab}}$ were not significantly perturbed when swapping the VK, the Ni-NTA interactions remained consistent with the KD measurements (Figure 4).

Interactions of Ni-NTA with Vк1 Q89 in the Pertuzumab VH5-

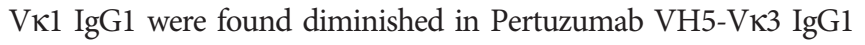
and abolished in the Pertuzumab VH5-Vk6 IgG1 (Figure S4). Interestingly, Vк1 Q89 formed another Q-stretch with the adjacent

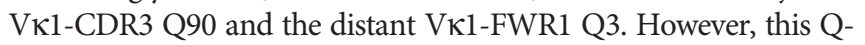

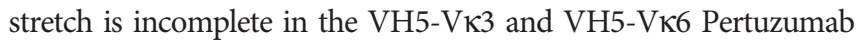
IgG1 variants due to the Q3V mutation in both Vк3 FWR1 and Vк6 FWR1 (Figure 5B), compromising the Ni-NTA interaction (Figure 4B). As in the Trastuzumab VH5-Vk1 IgE, residue H67 was not involved in all three Trastuzumab VH5-Vks IgG1 variants.

\section{Binding of Metal lons}

Measuring the interactions with various metal ions $\mathrm{Cu}^{2+}$ and $\mathrm{Co}^{2+}$ recharged onto the NTA sensors, Pertuzumab VH5-Vк1 IgE bound the other metals in the order of $\mathrm{Cu}^{2+}>\mathrm{Ni}^{2+}>\mathrm{Co}^{2+}$ (Figure 6A) as per Irving-Williams' order (50). From the analysis of protein-metal complexes $\left(1,409\right.$ for $\mathrm{Cu}^{2+} ; 1,810$ for $\mathrm{Ni}^{2+}$; and 716 for $\mathrm{Co}^{2+}$ in PDB, accessed in June 2020), histidine expectedly bound multiple metals, followed by aspartate, glutamate, cysteine, methionine, serine, and glutamine (Figure 6B). Only a few of these complexes are immunerelated $(12,26$, and 14 complexes with the metal ions, Figure 6B, small offset) and histidine was shown as the main residue interacting with all the three metal ions while glutamine bound only $\mathrm{Ni}^{2+}$. Notably, three protein- $\mathrm{Ni}^{2+}$ complexes (a single $\mathrm{VHH}$ antibody domain, PDB: 4PPT, and two HLA-peptide complexes, PDB: 5IB4 and 6EI2, shown in Figure 6C) contained both histidine and glutamine in their $\mathrm{Ni}^{2+}$ binding regions where glutamine either directly weakly interacted (4PPT) or was within close proximity to the $\mathrm{Ni}^{2+}$ binding histidine (5IB4 and 6EI2).

\section{DISCUSSION}

Through unsuccessful attempts to measure IgE interaction with His-tagged FceRI $\alpha$ via protein L immobilization (Figure S5) 
A

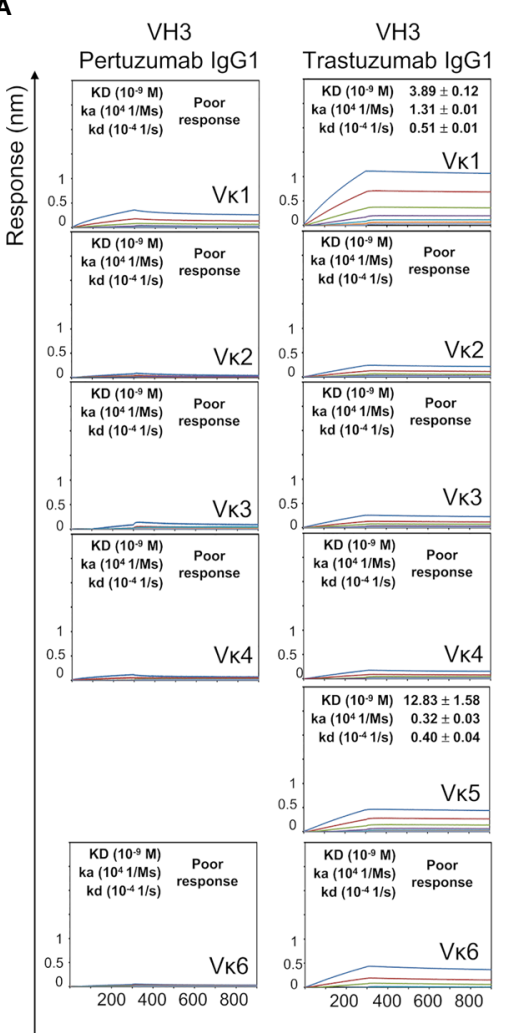

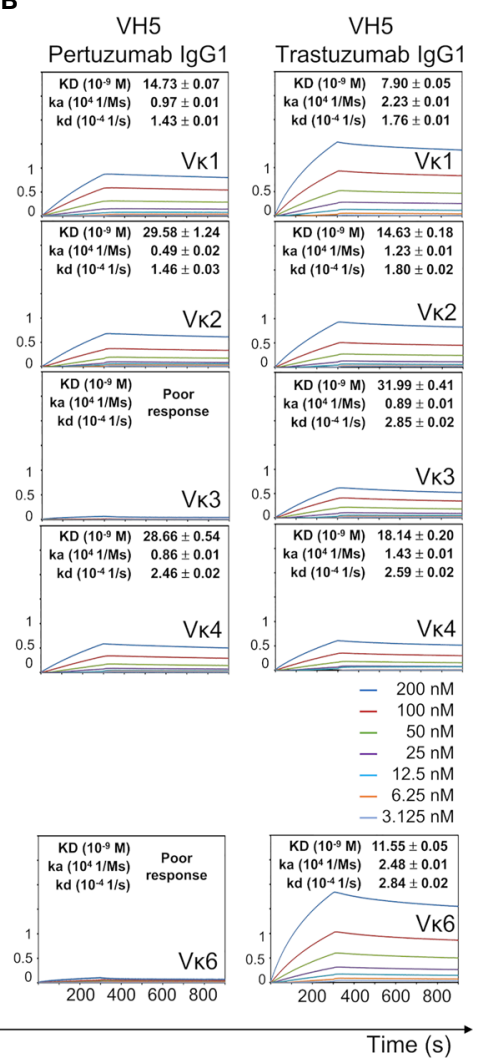

FIGURE 4 | Ni-NTA dissociation equilibrium constants (KD) of Pertuzumab and Trastuzumab VH3 (A) and VH5 (B) lgG1 variants paired with different Vks. The experiments were performed at concentrations from $200 \mathrm{nM}$ to $3.125 \mathrm{nM}$ of the antibodies. Values of KD (M), ka (1/Ms), and kd (1/s) were determined using the Octet RED96 system. The X-axis depicts the time (in seconds) while the Y-axis depicts the binding responses (nm). All the experiments were conducted in at least duplicates. "Poor response" reflects measurement responses that are less than $0.1 \mathrm{~nm}$ for at least three concentrations. Variants that could not be produced are left empty.

thereby eventually utilizing Ni-NTA biosensors, we found that Pertuzumab VH5 IgE interacted uniquely with Ni-NTA immobilized FceRI $\alpha$-His (30). The conclusion of $\mathrm{VH}$ influencing Fc-FceRI $\alpha$ engagement of the work remained valid due to (i) the similar KD of Trastuzumab VH3, VH5, and VH7 IgE variants to Ni-NTA (Figure 1A) but with varying KDs to FceRI $\alpha$-His (30), and (ii) the lack of Ni effects on Pertuzumab VH6 IgE binding Ni-NTA FceRI $\alpha$-His (present study). With respect to VH effects on the FcR engagement, we show here that the $\mathrm{VH}$ of IgEs can also engage $\mathrm{Ni}$ without compromising antigen recognition.

Systematic displacement of the FWRs and CDRs in Pertuzumab and Trastuzumab IgE and IgG1 revealed contribution of these regions to $\mathrm{Ni}^{2+}$ engagement. Trastuzumab variants (except for VH5-Vк1 IgE) bound better to the Ni-NTA when compared to the Pertuzumab IgE and IgG1. Further experiments with other heavy metals and NTA alone showed the interaction with Ni to be from Q-stretches. Since Trastuzumab and Pertuzumab are clinical therapeutics, the holistic (27) analysis of antibodies here clearly caution for sagacious design of biologics (51) to avoid possible interactions that may cause side effects.

Swapping the Ce (IgE) to $\mathrm{C} \gamma$ (IgG1) failed to restore the $\mathrm{Ni}^{2+}$ binding (completely abolished in Pertuzumab VH3 IgE) demonstrating the distal effects of the $\mathrm{CH}$. Despite possessing the known metal-binding histidine cluster "HEALHNH" (43) in C $\gamma$, there was no notable Ni engagement in most of the Pertuzumab VHs IgG1 variants, consistent with previous findings (17).

Of the IMGT VH/V $\kappa$ families and germlines sequences, only VH3, VH4, Vא1 (Tables S3 and S4), and VH5 frameworks had an extra histidine, yet this is only a portion of the overall antibody population (52). Since IgE is in lower quantities than IgGs in blood (53), nickel binding IgEs due to the extra histidine is of very small odds.

The modulation of Ni-binding by differential VK pairing while retaining histidine content showed the contribution of the $\mathrm{V}$ - and C- regions of the IgE variants in the structural formation of $\mathrm{Ni}$ binding Q-stretches (continuous clusters of at least 3 glutamines). These Q-stretches occurred at the heavy and light chain interface of the Trastuzumab IgE variants or at scaffolds between Vк1 FWR1 and CDR3 of the Pertuzumab VH5 IgG1 variants. Since many of our IgE variants exhibited better $\mathrm{Ni}$ binding than their IgG1 counterparts, the phenomenon is more prevalent in IgEs than IgGs.

Hapten-specific T cells (2) showed that $\mathrm{Ni}^{2+}$ bound MHCpeptide complexes have $\mathrm{Ni}^{2+}$ bridges between the histidine on the HLA-DR $\beta$-chain and the TCR $\alpha$ chain hypervariable region 


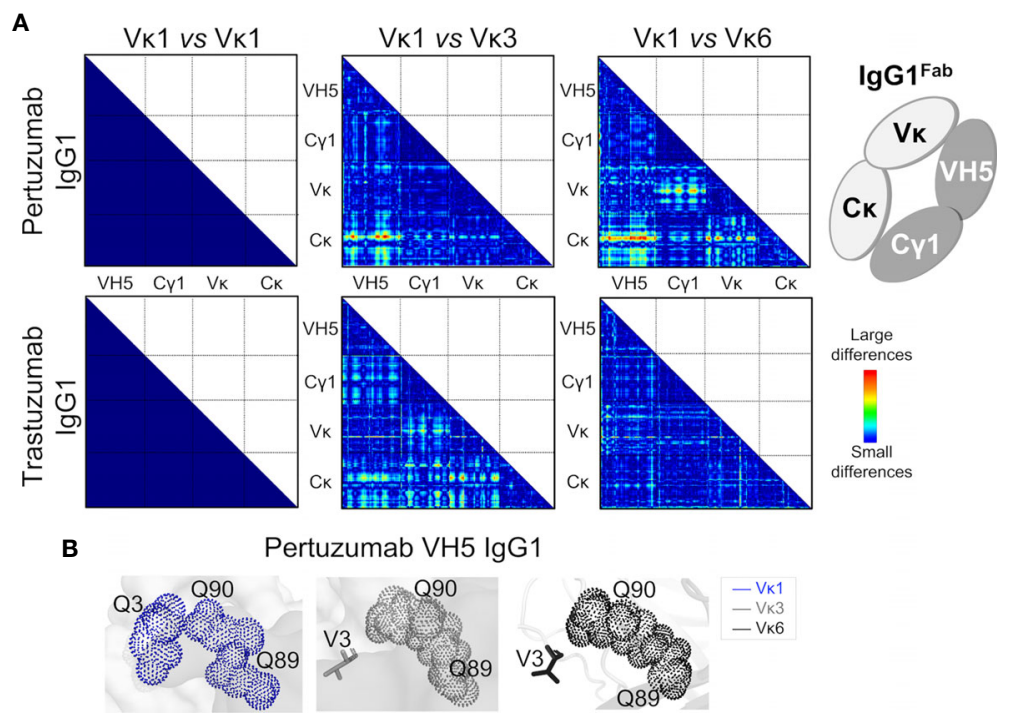

FIGURE 5 | Effects of the different VH5-Vк pairings in the Pertuzumab and Trastuzumab lgG1 variants. (A) Distance-based difference maps [generated using

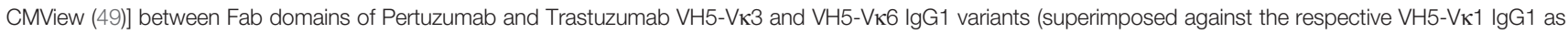
the reference) indicates more disruption by the $\bigvee_{\kappa} 3$ or $\bigvee_{\kappa} 6$ for Pertuzumab VH5 lgG1 variants, leading to varying changes in the heavy and light chain pairing. (B) An

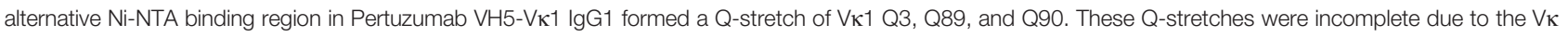

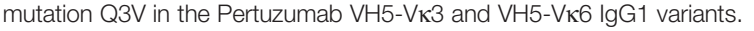

(14). Notwithstanding the $\mathrm{Ni}^{2+}$ engagement of the rheumatoid associated (15) peptide-bound HLA-B ${ }^{\star} 27: 05$ complex (PDB: 5IB4), we did not find other evidence. This may be because the reported peptide underwent an $\mathrm{Ni}^{2+}$ induced reorientation via an energetically driven mechanism congruent to the $\mathrm{Ni}^{2+}$ binding of the H67 in Pertuzumab but not Trastuzumab VH5-Vk1 IgE.

While metal binding can influence antigen presentation of both MHC class I and II $(15,44)$, the atypical non-antigenic mechanism of $\mathrm{Ni}^{2+}$ here can directly activate both TCR in Type IV or cross-link IgE on mast cells to trigger Type I (11) hypersensitivities. While linked recognition may occur in patients with dual clinical manifestations (4), it is extremely rare. Nonetheless, the mechanism in the IgEs was shown to be of non-conventional antigenic binding and more like that of antibody superantigens proteins $\mathrm{A}$ and $\mathrm{L}$ to be not-involving CDRs alone. Thus we propose the inclusion of nickel as new dual superantigen-like molecule if not a superantigen.

We note that the classification of superantigen and superantigen-like molecules to be very contentions with some in the immunology recognizing T-cell superantigens and not that of antibodies, and that nickel was excluded as a TCR superantigen $(14,35)$, However, molecular mechanisms that we uncover here may underlie why "specific combination of $\alpha$ and $\beta$-chains" are required for Ni-binding by TCRs (35) to form the Ni-binding amino acid stretches required as in our IgEs here.

Although we are unable to clinically validate the potential of the mechanism here underlying the cause of type I and IV nickel allergies given that access to a rare patient co-manifesting both nickel allergies is challenging, much less finding the right $\mathrm{T}$ - and B-cell clones in the patient, we have demonstrated that the phenomenon is possible with clinical therapeutics. The mechanism can underlie the metal-protein interaction reported in the two allergy types $(11,54)$ where natural VDJ generated antibodies and therapeutic antibodies could possess unintended Q-stretches. Such unintended nickel binding by antibodies can go undetected unless specifically looked for given the uncompromised intended antigen interaction (in our case Her2). Such a mechanism can underlie the dermatological side effects (19-21) associated with some biologics therapy and why prolonged exposure to nickel can elicit type I and IV hypersensitivities in later life akin to allergic rhinitis and Staphylococcus aureus colonization via protein A (55-58). Given that nickel is ubiquitous in modern day living (1), the selection of such IgEs or even T-cells is certainly a high possibility.

With previous evidence that some VH5 IgEs can exhibit prolonged interactions with FceRI $\alpha$ (30), thereby likely becoming the more dominant $\mathrm{VH}$ family found in asthmatics (59), the ability to bind to nickel in our VH5 IgEs seem to dovetail the rise of the otherwise rare nickel Type I allergy. Together, these findings demonstrate the importance of VH5 biased IgEs to which the actual discovery in allergic patients is necessary. The rarity of this may be attributed to the low percentage of IgE to total Ig, that together with the lower usage of VH5 compared to the other VH families of 1, 3, 4, and 6 (60), explain why type I nickel allergies are not more prevalent. However, given that the phenomenon can occur with VH3IgEs, it is prudent to minimize unnecessary exposure to nickel and other metal as with that of other superantigens.

As the binding of $\mathrm{Ni}$ occurs at the $\mathrm{V}$-regions, the FcR engagements are unlikely to be affected. Thus, the IgG 


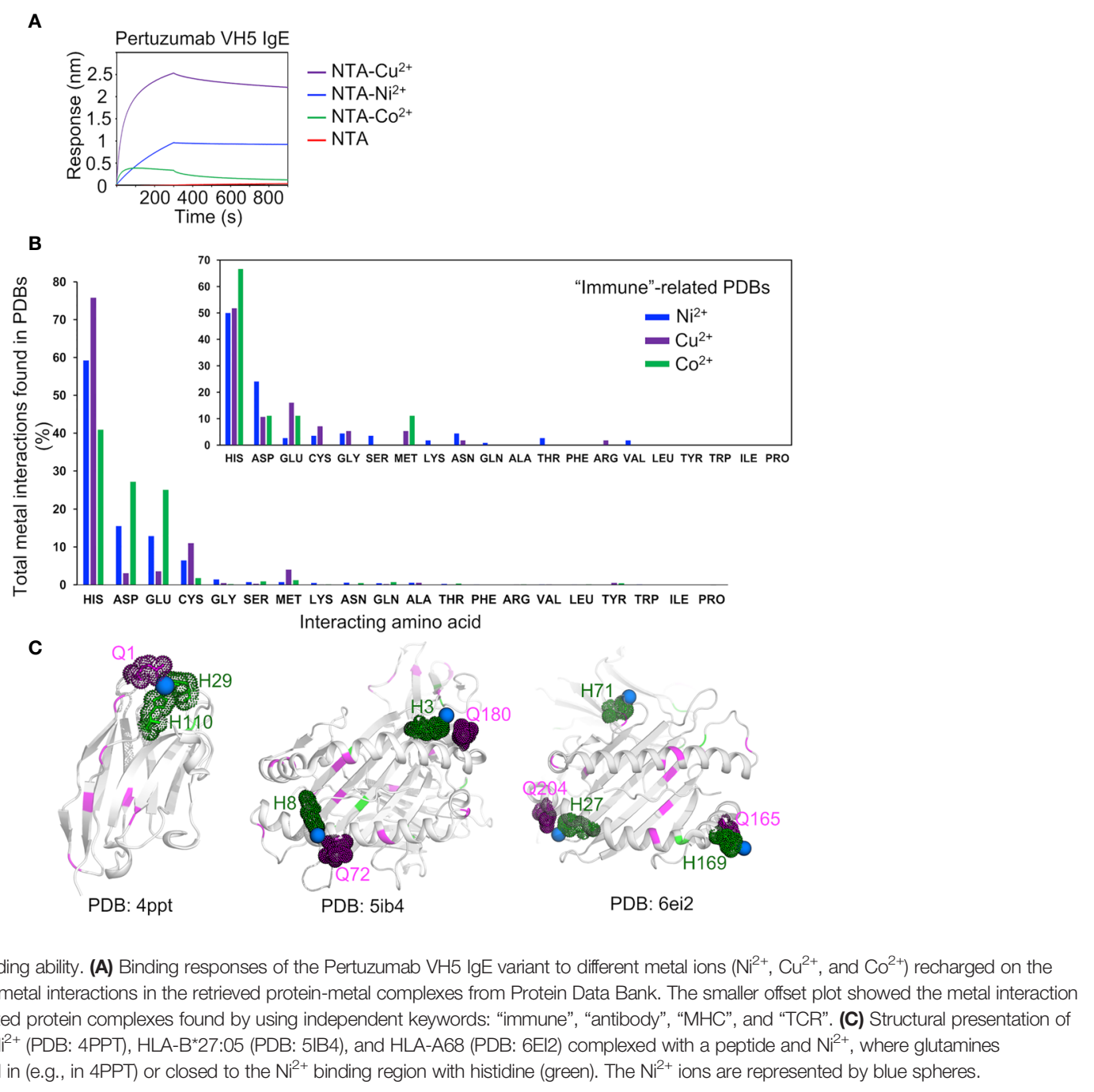

counterpart may function to mop up Ni-protein complexes that may otherwise cross-link sensitized mast cells or moderate the activation of dendritic cells/keratinocytes for detoxification (61). Further studies of Ni-specific antibody isotype levels in patients would be necessary to investigate this.

Since Ni can act as adjuvants to other metals (62), there are possible exploitation of the mechanism to engineer better antibodies similar to the Bi-specific T-cell engagers (BiTEs) technology where nickel binding may attract associated TCRs. Further tailoring $\mathrm{Ni}$ towards such uses is required, with mindfulness of inducing Ni hypersensitivity and its effects.

In conclusion from our systemic displacement of antibody regions, Ni-binding antibodies were found to do so via a combination of factors involving the CDRs and FWRs of both $\mathrm{VH}$ and $\mathrm{V} \kappa$ chains, and the $\mathrm{CH}$ to form inducible structural Qstretches without losing antigen specificity. This superantigenlike property can be exploited in potential therapeutic interventions as well as in biotechnological purification and antibody engineering purposes.

\section{DATA AVAILABILITY STATEMENT}

The original contributions presented in the study are included in the article/Supplementary Material. Further inquiries can be directed to the corresponding author.

\section{AUTHOR CONTRIBUTIONS}

WHL, WLL, and JJP produced the recombinant antibodies and performed the biolayer interferometry experiments. JYY performed the framework germline experiment. CTTS performed the computational simulation and analyses. CTTS, WHL, and SKEG analysed the results and wrote the manuscript. SKEG designed and supervised all aspect of the study. All authors contributed to the article and approved the submitted version.

\section{FUNDING}

The work was supported by $A^{\star}$ STAR core funds. 


\section{ACKNOWLEDGMENTS}

The work was supported by A*STAR. We thank Chan KwokFong for making the augmented reality (AR) presentation for Figure 2. SG thanks Ebenezer for the help rendered.

\section{REFERENCES}

1. Flyvholm MA, Nielsen GD, Andersen A. Nickel Content of Food and Estimation of Dietary Intake. Z Lebensm Unters Forsch (1984) 179(6):42731. doi: 10.1007/BF01043419

2. Saito M, Arakaki R, Yamada A, Tsunematsu T, Kudo Y, Ishimaru N. Molecular Mechanisms of Nickel Allergy. Int J Mol Sci (2016) 17(2):202. doi: 10.3390/ijms17020202

3. Ahlström MG, Thyssen JP, Wennervaldt M, Menné T, Johansen JD. Nickel Allergy and Allergic Contact Dermatitis: A Clinical Review of Immunology, Epidemiology, Exposure, and Treatment. Contact Dermatitis (2019) 81 (4):227-41. doi: 10.1111/cod.13327

4. Walsh ML, Smith VH, King CM. Type I and Type IV Hypersensitivity to Nickel. Aust J Dermatol (2010) 51:285-6. doi: 10.1111/j.1440-0960. 2010.00664.x

5. Gelardi M, Guarino R, Taliente S, Quaranta N, Carperntieri A, Passalacqua G. Allergic and Nonallergic Rhinitis and Skin Sensitization to Metals: Is There a Link? Eur Ann Allergy Clin Immunol (2017) 49(3):106-9.

6. Kolberg L, Forster F, Gerlich J, Weinmayr G, Genuneit J, Windstetter D, et al. Nickel Allergy Is Associated With Wheezing and Asthma in Cohort of Young German Adults: Results From the SOLAR Study. ERJ Open Res (2020) 6:00178-2019. doi: 10.1183/23120541.00178-2019

7. Ganju RK, Smiley ST, Bajorath J, Novotny J, Reinherz EL. Similarity Between Fluorescein-Specific T-Cell Receptor and Antibody in Chemical Details of Antigen Recognition. PNAS (1992) 89(23):11552-6. doi: 10.1073/pnas. 89.23.11552

8. Dunbar J, Knapp B, Fuchs A, Shi J, Deane CM. Examining Variable Domain Orientations in Antigen Receptors Gives Insight Into TCR-Like Antibody Design. PloS Comput Biol (2014) 10(9):e1003852. doi: 10.1371/ journal.pcbi.1003852

9. Wong WK, Leem J, Deane CM. Comparative Analysis of the CDR Loops of Antigen Receptors. Front Immunol (2019) 10:2454. doi: 10.3389/ fimmu.2019.02454

10. Thierse H-J, Moulon C, Allespach Y, Zimmermann B, Doetze A, Kuppig S, et al. Metal-Protein Complex-Mediated Transport and Delivery of $\mathrm{Ni2}+$ to TCR/MHC Contact Sites in Nickel-Specific Human T Cell Activation. J Immunol (2004) 172(3):1926-34. doi: 10.4049/jimmunol.172.3.1926

11. Sastre J, Fernández-Nieto M, Marañón F, Fernandez-Caldas E, Pelta R, Quirce S. Allergenic Cross-Reactivity Between Nickel and Chromium Salts in Electroplating-Induced Asthma. J Allergy Clin Immunol (2001) 108(4):650-1. doi: 10.1067/mai.2001.118294

12. Janeway C, Travers P, Walport M, Shlomchik M. Immunobiology: The Immune System in Health and Disease. 5th. New York: Garland Science (2001).

13. Sudan RJJ, Kumari JLJ CS. Ab Initio Coordination Chemistry for Nickel Chelation Motifs. PloS One (2015) 10(5):e0126787. doi: 10.1371/ journal.pone. 0126787

14. Gamerdinger K, Moulon C, Karp DR, Bergen JV, Koning F, Wild D, et al. A New Type of Metal Recognition by Human T Cells: Contact Residues for Peptide-Independent Bridging of T Cell Receptor and Major Histocompatibility Complex by Nickel. J Exp Med (2003) 197(10):1345-53. doi: $10.1084 /$ jem.20030121

15. Driller R, Ballaschk M, Schmieder P, Uchanska-Ziegler B, Ziegler A, Loll B. Metal-Triggered Conformational Reorientation of a Self-Peptide Bound to a Disease-Associated HLA-B²7 Subtype. J Biol Chem (2019) 294(36):1326979. doi: 10.1074/jbc.RA119.008937

16. Zeng Y-B, Zhang D-M, Li H, Sun H. Binding of Ni2+ to a Histidine- and Glutamine-Rich Protein, Hpn-Like. J Bio Inorg Chem (2008) 13(7):1121-31. doi: 10.1007/s00775-008-0397-0

\section{SUPPLEMENTARY MATERIAL}

The Supplementary Material for this article can be found online at: https://www.frontiersin.org/articles/10.3389/fimmu.2021. 676048/full\#supplementary-material

17. Rosen CB, Kodal ALB, Nielsen JS, Schaffert DH, Scavenius C, Okholm AH, et al. Template-Directed Covalent Conjugation of DNA to Native Antibodies, Transferrin and Other Metal-Binding Proteins. Nat Chem (2014) 6(9):804-9. doi: 10.1038/nchem.2003

18. Aparicio-Soto M, Riedel F, Leddermann M, Bacher P, Scheffold A, Kuhl H, et al. TCRs With Segment TRAV9-2 or a CDR3 Histidine are Overrepresented Among Nickel-Specific CD4+ T Cells. Allergy (2020) 00:1-13. doi: 10.1111/ all.14322

19. Skin Reactions to Targeted Therapy and Immunotherapy. American Society of Clinical Oncology (ASCO) (2019). Available at: https://www.cancer.net/ coping-with-cancer/physical-emotional-and-social-effects-cancer/managingphysical-side-effects/skin-reactions-targeted-therapy-and-immunotherapy.

20. Pasadyn SR, Knabel D, Fernandez AP, Warren CB. Cutaneous Adverse Effects of Biologic Medications. Cleveland Clinic J Med (2020) 87(5):288-99. doi: 10.3949/ccjm.87a.19119

21. Sehgal R, Stratman EJ, Cutlan JE. Biologic Agent-Associated Cutaneous Adverse Events: A Single Center Experience. Clin Med Res (2018) 16(12):41-6. doi: $10.3121 / \mathrm{cmr} .2017 .1364$

22. Hedberg YS, Dobryden I, Chaudhary H, Wei Z, Claesson PM, Lendel C. Synergistic Effects of Metal-Induced Aggregation of Human Serum Albumin. Colloids Surf B: Biointerf (2019) 173:751-8. doi: 10.1016/j.colsurfb. 2018.10.061

23. Moussa EM, Panchal JP, Moorthy BS, Blum JS, Joubert MK, Narhi LO, et al. Immunogenicity of Therapeutic Protein Aggregates. J Pharm Sci (2016) 105 (2):417-30. doi: 10.1016/j.xphs.2015.11.002

24. Kurt OK, Basaran N. Occupational Exposure to Metals and Solvents: Allery and Airway Diseases. Curr Allergy Asthma Rep (2020) 20(8):38. doi: 10.1007/ s11882-020-00931-7

25. Tiotiu AI, Novakova S, Labor M, Emelyanov A, Mihaicuta S, Novakova P, et al. Progress in Occupational Asthma. Int J Environ Res Public Health (2020) 17(12):4554. doi: 10.3390/ijerph17124553

26. Malo JL, Cartier A, Gagnon G, Evans SL, Dolovich J. Isolated Late Asthmatic Reaction Due to Nickel Sulphate Without Antibodies to Nickel. Clin Allergy (1985) 15(2):95-9. doi: 10.1111/j.1365-2222.1985.tb02261.x

27. Phua SX, Chan KF, Su CTT, Poh JJ, Gan SKE. Perspective: The Promises of a Holistic View of Proteins - Impact on Antibody Engineering and Drug Discovery. Biosci Rep (2019) 39(1):pii: BSR20181958. doi: 10.1042/ BSR20181958

28. Su CTT, Lua WH, Ling WL, Gan SKE. Allosteric Effects Between the Antibody Constant and Variable Regions: A Study of IgA Fc Mutations on Antigen Binding. Antibodies (2018) 7(2):20. doi: 10.3390/antib7020020

29. Lua WH, Ling WL, Yeo JY, Poh JJ, Lane DP, Gan SKE. The Effects of Antibody Engineering $\mathrm{CH}$ and $\mathrm{CL}$ in Trastuzumab and Pertuzumab Recombinant Models: Impact on Antibody Production and AntigenBinding. Sci Rep (2018) 8(1):718. doi: 10.1038/s41598-017-18892-9

30. Lua WH, Su CTT, Yeo JY, Poh JJ, Ling WL, Phua SX, et al. Role of the IgE Variable Heavy Chain in Fcerio and Superantigen Binding in Allergy and Immunotherapy. J Allergy Clin Immunol (2019) 144(2):514-23. doi: 10.1016/ j.jaci.2019.03.028

31. Ling WL, Lua WH, Poh JJ, Yeo JY, Lane DP, Gan SKE. Effect of VH-VL Families in Pertuzumab and Trastuzumab Recombinant Production, Her2 and Fcriia Binding. Front Immunol (2018) 9:469. doi: 10.3389/ fimmu.2018.00469

32. Guiducci S, Giacomelli R, Tyndall A, Matucci Cerinic M. Infection and Systematic Sclerosis. Infect Autoimmun (2004) 613-22. doi: 10.1016/B978044451271-0.50047-8

33. Levinson AI, Kozlowski L, Zheng Y, Wheatley L. B-Cell Superantigens: Definition and Potential Impact on the Immune Response. J Clin Immunol (1995) 15:S26-36. doi: 10.1007/BF01540891 
34. Genovese A, Bouvet J-P, Florio G, Lamparter-Schummert B, Bjorck L, Marone G. Bacterial Immunoglobulin Superantigen Proteins A and L Activate Human Heart Mast Cells by Interacting With Immunoglobulin E. Infect Immun (2000) 68(10):5517-24. doi: 10.1128/IAI.68.10.5517-5524.2000

35. Vollmer J, Weltzien HU, Moulon C. TCR Reactivity in Human Nickel Allergy Indicates Contacts With Complementarity-Determining Region 3 But Excludes Superantigen-Like Recognition. J Immunol (1999) 163 (5):2723-31.

36. Su CTT, Ling WL, Lua WH, Poh JJ, Gan SKE. The Role of Antibody Vк Framework 3 Region Towards Antigen Binding: Effects on Recombinant Production and Protein L Binding. Sci Rep (2017) 7:3766. doi: 10.1038/ s41598-017-02756-3

37. Abraham MJ, Murtola T, Schulz R, Pall S, Smith JC, Hess B, et al. GROMACS: High Performance Molecular Simulations Through Multi-Level Parallelism From Laptops to Supercomputers. SoftwareX (2020) 1-2:19-25. doi: 10.1016/ j.softx.2015.06.001

38. Case DA, Berryman JT, Betz RM, Cerutti DS, T.E. Cheatham I, Darden TA, et al. AMBER 14. San Francisco: University of California (2015).

39. Pettersen E, Goddard T, Huang C, Couch G, Greenblatt D, Meng E, et al. UCSF Chimera - A Visualization System for Exploratory Research and Analysis. J Comput Chem (2004) 25(13):1605-12. doi: 10.1002/jcc.20084

40. Trott O, Olson AJ. AutoDock Vina: Improving the Speed and Accuracy of Docking With a New Scoring Function, Efficient Optimization and Multithreading. J Comput Chem (2010) 31:455-61. doi: 10.1002/jcc.21334

41. Cimermancic P, Weinkam P, Rettenmaier TJ, Bichmann L, Keedy DA, Woldeyes RA, et al. CryptoSite: Explanding the Druggable Proteome by Charaterization and Prediction of Cryptic Binding Sites. J Mol Biol (2016) 428(4):709-19. doi: 10.1016/j.jmb.2016.01.029

42. Schmidtke P, Guilloux VL, Maupetit J, Tuffery P. Fpocket: Online Tools for Protein Ensemble Pocket Detection and Tracking. Nucleic Acids Res (2010) 38 (Web Server issue):W582-9. doi: 10.1093/nar/gkq383

43. Hale JE, Beidler DE. Purification of Humanized Murine and Murine Monoclonal Antibodies Using Immobilized Metal-Affinity Chromatography. Anal Biochem (1994) 222(1):29-33. doi: 10.1006/ abio.1994.1449

44. Wang Y, Dai S. Structural Basis of Metal Hypersensitivity. Immunol Res (2013) 55(0):83-90. doi: 10.1007/s12026-012-8351-1

45. Fanning SW, Walter R, Horn JR. Structural Basis of an Engineered DualSpecific Antibody: Conformational Diversity Leads to a Hypervariable Loop Metal-Binding Site. Protein Eng Des Sel (2014) 27(10):391-7. doi: 10.1093/ protein/gzu033

46. Schrodinger L. The PyMOL Molecular Graphics System. Version 2.2.2 Ed. (2019).

47. Poh JJ, Phua SX, Chan KF, Gan SKE. Commentary: Augmented Reality Scientific Phone Apps - Making the APD AR Holistic Review App and Using Existing AR Apps for Scientific Publications. Sci Phone Apps Mobile Dev (2018) 4:4. doi: 10.30943/2018/28092018

48. Chan K-F, Poh J-J, Wu W-L, Gan SK-E. Augmented Reality in Scientific Visualization and Communications: A New Dawn of Looking at Antibody Interactions. Antibody Ther (2020) 3(3):221-6. doi: 10.1093/abt/tbaa021

49. Vehlow C, Stehr H, Winkelmann M, Duarte JM, Petzold L, Dinse J, et al. CMView: Interactive Contact Map Visualization and Analysis. Bioinformatics (2011) 27(11):1573-4. doi: 10.1093/bioinformatics/btr163

50. Irving H, Williams RJP. The Stability of Transition-Metal Complexes. J Chem Soc (Resumed) (1953) 0:3192-210. doi: 10.1039/JR9530003192
51. Ling W-L, Lua W-H, Gan SK-E. Sagacity in Antibody Humanization for Therapeutics, Diagnostics and Research Purposes: Considerations of Antibody Elements and Their Roles. Antibody Ther (2020) 3(2):71-9. doi: $10.1093 / \mathrm{abt} / \mathrm{tbaa} 005$

52. Tiller T, Schuster I, Deppe D, Siegers K, Strohner R, Herrmann T, et al. A Fully Synthetic Human Fab Antibody Library Based on Fixed VH/VL Framework Pairings With Favorable Biophysical Properties. MAbs (2013) 5(3):445-70. doi: $10.4161 / \mathrm{mabs} .24218$

53. Gould HJ, Sutton BJ, Beavil AJ, Beavil RL, McCloskey N, Coker HA, et al. The Biology of IgE and the Basis of Allergic Disease. Annu Rev Immunol (2003) 21:579-628. doi: 10.1146/annurev.immunol.21.120601.141103

54. Bright P, Burge P, O'Hickey S, Gannon P, Robertson A, Boran A. Occupational Asthma Due to Chrome and Nickel Electroplating. Thorax (1997) 52(1):28-32. doi: 10.1136/thx.52.1.28

55. Peschel A, Sahl H-G. The Co-Evolution of Host Cationic Antimicrobial Peptides and Microbial Resistance. Nat Rev Microbiol (2006) 4(7):529-36. doi: $10.1038 /$ nrmicrol441

56. Cole AL, Schmidt-Owens M, Beavis AC, Chong CF, Tarwater PM, Schaus J, et al. Cessation From Smoking Improves Innate Host Defense and Clearance of Experimentally Inoculated Nasal Staphylococcus Aureus. Infect Immun (2018) 86(4):e00912-17. doi: 10.1128/IAI.00912-17

57. Sun Y, Emolo C, Holtfreter S, Wiles S, Kreiswirth B, Missiakas D, et al. Staphylococcal Protein A Contributes to Persistent Colonization of Mice With Staphylococcus Aureus. J Bacteriol (2018) 200(9):e00735-17. doi: 10.1128/JB.00735-17

58. Sakr A, Bregeon F, Mege J-L, Rolain J-M, Blin O. Staphylococcus Aureus Nasal Colonization: An Update on Mechanisms, Epidemiology, Risk Factors, and Subsequent Infections. Front Microbiol (2018) 9:2419. doi: 10.3389/ fmicb.2018.02419

59. Snow RE, Chapman CJ, Frew AJ, Holgate ST, Stevenson FK. Analysis of Ig VH Region Genes Encoding IgE Antibodies in Splenic B Lymphocytes of a Patient With Asthma. J Immunol (1995) 154(10):5576-81.

60. Kohsaka H, Carson DA, Rassenti LZ, Ollier WE, Chen PP, Kipps TJ, et al. The Human Immunoglobulin $\mathrm{V}(\mathrm{H})$ Gene Repertoire is Genetically Controlled and Unaltered by Chronic Autoimmune Stimulation. J Clin Invest (1996) 98 (12):2794-800. doi: 10.1172/JCI119106

61. Hennen J, Aeby P, Goebel C, Schettgen T, Oberli A, Kalmes M, et al. Cross Talk Between Keratinocytes and Dendritic Cells: Impact on the Prediction of Sensitization. Toxicol Sci (2011) 123(2):501-10. doi: 10.1093/toxsci/kfr174

62. Bonefeld CM, Nielsen MM, Vennegaard MT, Johansen JD, Geisler C, Thyssen JP. Nickel Acts as an Adjuvant During Cobalt Sensitization. Exp Dermatol (2015) 24(3):229-31. doi: 10.111111/exd.12634

Conflict of Interest: SKEG was employed by APD SKEG Pte Ltd., Singapore, Singapore.

The remaining authors declare that the research was conducted in the absence of any commercial or financial relationships that could be construed as a potential conflict of interest.

Copyright (c) 2021 Su, Lua, Poh, Ling, Yeo and Gan. This is an open-access article distributed under the terms of the Creative Commons Attribution License (CC BY). The use, distribution or reproduction in other forums is permitted, provided the original author(s) and the copyright owner(s) are credited and that the original publication in this journal is cited, in accordance with accepted academic practice. No use, distribution or reproduction is permitted which does not comply with these terms. 